\title{
Pancreatic head carcinoma and vascular endothelial growth factor (VEGF-A) concentration in portal blood: its association with cancer grade, tumor size and probably poor prognosis
}

\author{
Piotr Hogendorf ${ }^{1}$, Adam Durczyński ${ }^{1}$, Anna Kumor ${ }^{2}$, Janusz Strzelczyk ${ }^{1}$
}

${ }^{1}$ Department of General and Transplant Surgery, Medical University of Lodz, Poland ${ }^{2}$ Department of Laboratory Diagnostics, Medical University of Lodz, Poland

Submitted: 3 December 2010

Accepted: 4 September 2011

Arch Med Sci 2014; 10, 2: 288-293

DOI: 10.5114/aoms.2014.42581

Copyright (c) 2014 Termedia \& Banach
Corresponding author: Piotr Hogendorf MD, PhD Department of General and Transplant Surgery Medical University of Lodz 22 Kopcińskiego St 90-153 Lodz, Poland Phone: +48 606699045 Fax: +48 426791091 E-mail: phog@wp.pl

\begin{abstract}
Introduction: Vascular endothelial growth factor (VEGF) is overexpressed in pancreatic cancer. Although VEGF has been shown to be a probable marker for poor prognosis, the VEGF concentration in portal blood has not yet been clinically reported in pancreatic ductal adenocarcinoma (PDAC). The aim of the study was to measure VEGF-A portal blood concentration in patients with PDAC and to evaluate its performance as a prognostic marker.

Material and methods: Thirty-six consecutive patients out of 57 operated on for pancreatic head lesion with pathologically verified diagnosis of PDAC were enrolled in this study. We evaluated the VEGF concentration in portal blood samples obtained intraoperatively and associated their values with tumor size, stage, grade and survival.

Results: The portal VEGF-A concentration was associated with tumor grade (G1: $80.52 \pm 43.05$ vs. G2: $185.39 \pm 134.98, p=0.006, \mathrm{G} 2: 185.39 \pm 134.98$ vs. G3: $356.46 \pm 229.12, p=0.08)$, and there was a positive correlation with tumor size $(r=0.42, p<0.05)$. In the multivariate regression analysis high levels of VEGF-A were not correlated with poor survival $(\mathrm{HR}=5.22,95 \% \mathrm{Cl}=$ -0.6457 to $3.9513, p=0.19$ ).

Conclusions: The portal VEGF-A concentration is associated with tumor grade and size. The correlation of portal VEGF-A with poor survival is not clear and needs further investigation.
\end{abstract}

Key words: pancreatic cancer, grade, surgery, vascular endothelial growth factor, vascular endothelial growth factor, survival.

\section{Introduction}

Pancreatic cancer is one of the most aggressive malignancies. Every year 34000 new cases of pancreatic ductal adenocarcinoma (PDAC) are reported in the United States. Very high mortality, which is nearly equal to the incidence rate, makes PDAC the fourth most common cause of cancer-related deaths [1]. The probability that a patient with newly diagnosed PDAC will be suitable for pancreatic resection is $10-20 \%$. The 5 -year survival rate after surgery is not higher than $7-32 \%$ [2, 3]. Various possible prognostic markers have been studied so far, including tumor size, tumor stage, lymph node status as well as molecular tumor markers [4] 
but only in peripheral blood or within the tumor mass. According to the literature this is the first study describing a prognostic factor in pancreatic cancer in intraoperatively acquired portal blood samples.

Angiogenesis plays a crucial role in tumor development. In order to grow bigger than $2-3 \mathrm{~mm}^{3}$ a tumor requires an additional network of blood vessels [5].

Vascular endothelial growth factor (VEGF) has been shown to be the major mediator in physiological and pathological angiogenesis [6]. The VEGF belongs to the platelet-derived growth factor supergene family (PDGF/VEGF). The VEGF family consists of at least 8 members: VEGF-A, VEGF-B, VEGF-C, VEGF-D, VEGF-E, VEGF-F, viral orf-VEGF and placental growth factor (PIGF) [7]. The most important role in the growth and maintenance of vessels from endothelial cells is played by the gene for VEGF-A. As the result of alternative splicing at least 7 different isoforms containing $121,145,148,165,183,189$, 206 amino acids have been identified so far. The dominant subtype of VEGF-A is VEGF-A ${ }_{165}$ according to biological activity as well as amount [8]. The overexpression of VEGF-A, VEGF-B, VEGF-C, VEGF-D in patients with pancreatic cancer has been proved $[9,10]$. Although VEGF has been shown to be a probable marker for poor prognosis in various tumors, as well as in PDAC [11-13], the results of some studies are controversial. The VEGF-A concentration in portal blood has not yet been clinically reported in pancreatic cancer. It is highly probable that levels of tumor produced markers could be higher in the portal vein. Portal blood samples are less prone to the effect of systemic blood dilution and inactivation of some markers in the liver.

Table I. Cancer staging (according to the International Union Against Cancer) and differentiation in the study group

\begin{tabular}{|lc|}
\hline Variable & Results \\
\hline Total portal blood samples & 36 \\
\hline Male & $20(56 \%)$ \\
\hline Female & $16(44 \%)$ \\
\hline Stage I & $0(0 \%)$ \\
\hline Stage III & $14(38.9 \%)$ \\
\hline Stage IV & $10(28.7 \%)$ \\
\hline Grade 1 & $11(30.6 \%)$ \\
\hline Grade 2 & $8(26.7 \%)$ \\
\hline Grade 3 & $18(36 \%)$ \\
\hline
\end{tabular}

\section{Material and methods}

There were 57 patients admitted to the Department of General and Transplant Surgery of Medical University of Lodz for the surgical treatment of newly diagnosed tumor in the pancreatic head or periampullary area. Patients who were diagnosed with pathology other than PDAC or were receiving drugs that could alter VEGF- $A_{165}$ measurement for less than 7 days before surgery, such as antiplatelet drugs or corticosteroids, were excluded from the study. Finally we enrolled 36 consecutive patients (20 males and 16 females, aged 48-76 years, mean 60.8 years) to this study. None of the patients received adjuvant chemoradiotherapy. All the patients underwent surgery - 22 standard pancreaticoduodenectomies, 12 doublebypass procedures and 2 gastroenterostomies were performed. In cases of nonresectable tumor, a pathological specimen (fine needle biopsy) was obtained during surgery (Tables I and II).

Following laparotomy and dissection of the hepatoduodenal ligament, the portal vein was identified and sampled. All samples were collected within 45 min from the skin incision. A blood sample of $9 \mathrm{ml}$ (EDTA) was obtained and centrifuged immediately at $3000 \mathrm{rpm}$ for $15 \mathrm{~min}$ at $4^{\circ} \mathrm{C}$. Plasma was stored at $-80^{\circ} \mathrm{C}$ until the day of analysis. The VEGF-A ${ }_{165}$ levels were determined using ELISA (R\&D Systems Inc., Minneapolis, MN), according to the manufacturer's instructions. The detection limit for human VEGF-A ${ }_{165}$ was $8 \mathrm{pg} / \mathrm{ml}$. All measurements were performed in duplicate, and no significant differences were found. We evaluated the VEGF- $A_{165}$ concentration in 36 intraoperatively obtained portal blood samples.

All the patients have been followed up for a period of 1-35 months.

All excised tumors were evaluated with standard pathological examinations, in accordance with the World Health Organization (WHO) criteria. In 31 out of 36 cases the grade of tumor was assessed in standard pathological diagnosis. The tumor size, stage and survival rate were also assessed. The tumor size was calculated according to Hotz as tumor volume $(\mathrm{V}=$ length $\times$ depth $\times$ width/2) [14].

Table II. Lymph nodes status and resection margins status in patients who underwent pancreaticoduodenectomy

\begin{tabular}{|lc|}
\hline Pancreaticoduodenectomy group & Results \\
\hline Positive lymph nodes & $12 / 22(55 \%)$ \\
\hline Resection R0 & $16 / 22(72 \%)$ \\
\hline Resection R1 & $6 / 22(27 \%)$ \\
\hline Resection R2 & $0 / 22(0 \%)$ \\
\hline
\end{tabular}




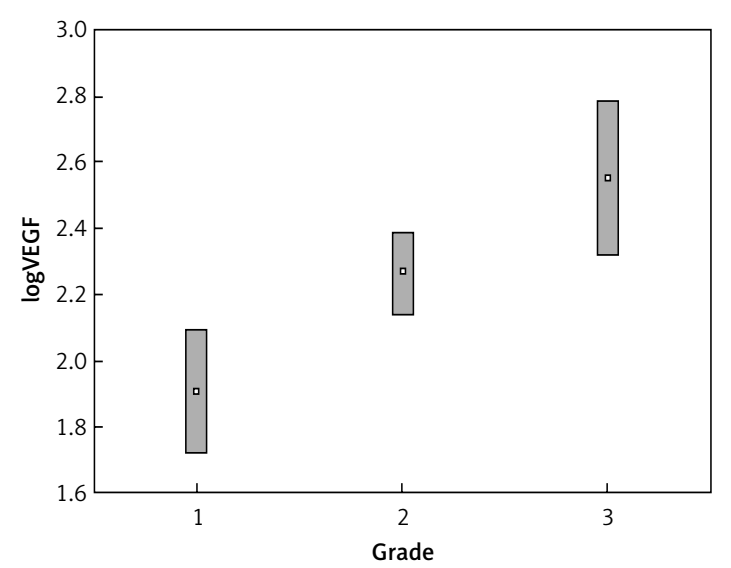

Figure 1. Portal vein VEGF-A ${ }_{165}$ levels (log VEGF-A ${ }_{165}$ ) and grade of the tumor. $P$-level between: $G 1$ vs. G2: $p=0.006 ; \mathrm{G} 2$ vs. G3: $p=0.08 ; \mathrm{G} 1$ vs. G3: $p=0.004$

This study was approved by the Bioethical Commission of the Medical University of Lodz.

\section{Statistical analysis}

Continuous variables are presented as mean \pm standard deviation and were compared using Student's $t$ test and the Mann-Whitney $U$ test. Multivariate analysis (ANOVA) with the Tukey test for differences between subgroups was used for the analysis of continuous variables in more than two groups. The survival was assessed with Kaplan-Meier survival curves. Survival rates for different groups were compared using the Gehan-Wilcoxon test for the Kaplan-Meier curve. Predictors for prognosis of the patients were assessed using Cox multiple

\section{A}

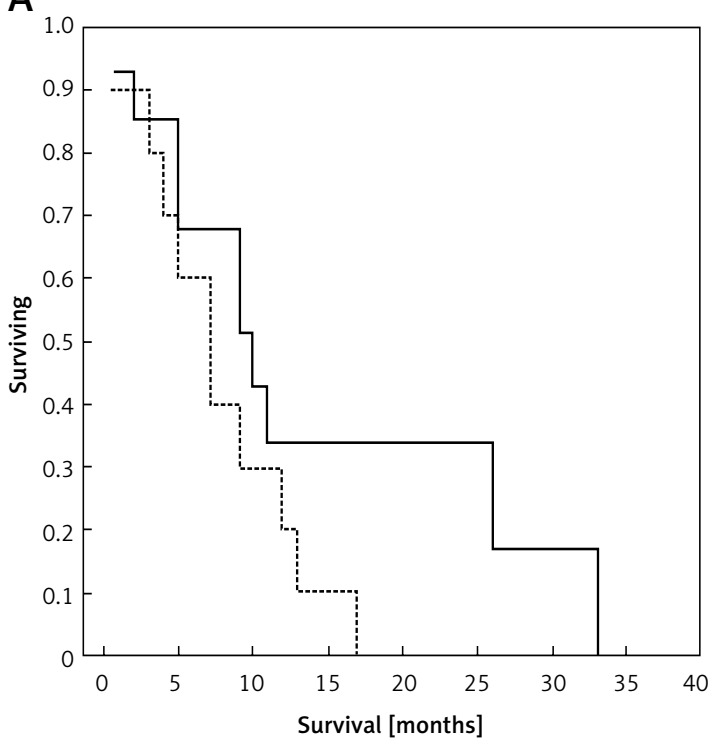

hazards regression analysis. Correlations were performed using a nonparametric (Spearman) correlation analysis. Value of $p<0.05$ was considered statistically significant. Statistical analysis was carried out using Statistica (StatSoft, Tulsa, USA).

\section{Results}

The median tumor volume was $4.5 \mathrm{~cm}^{3}$ (IQR: 2-8.75 $\left.\mathrm{cm}^{3}\right)$. There was no association between the stage of the disease and portal VEGF-A $\mathrm{A}_{165}$ concentration ( $T=2$ median: 158.4, IQR: 117.3-230.3).

There was no correlation either between the portal VEGF-A $A_{165}$ level and age $\left(\log \left(\right.\right.$ VEGF-A $\left._{165}\right), R=$ $-0.11, p=0.43$. The portal VEGF-A $A_{165}$ concentration was associated with tumor grade (G1: $80.52 \pm 43.05$ vs. G2: $185.39 \pm 134.98, p=0.006, \mathrm{G} 2: 185.39$ \pm 134.98 vs. $\mathrm{G} 3: 356.46 \pm 229.12, p=0.08$ ) (Figure 1 ). In the multivariate regression analysis, high levels of VEGF-A ${ }_{165}$ were not associated with poor survival with statistical significance $(\mathrm{HR}=5.22,95 \% \mathrm{Cl}=$ -0.6457 to $3.9513, p=0.16)$.

The group of patients with higher than median VEGF-A ${ }_{165}$ level $(150.51 \mathrm{ng} / \mathrm{ml})$ probably has lower median survival than the group with lower VEGF-A $A_{165}$ level $(p=0.12)$ (Figure $2 \mathrm{~A}$ ) but to achieve statistical significance a larger group of patients is required. The VEGF-A ${ }_{165}$ level was also correlated with tumor size (positive correlation $R=0.42, p<0.05)$.

\section{Discussion}

The VEGF plays a crucial role in tumor angiogenesis. In an autocrine and paracrine manner VEGF

B

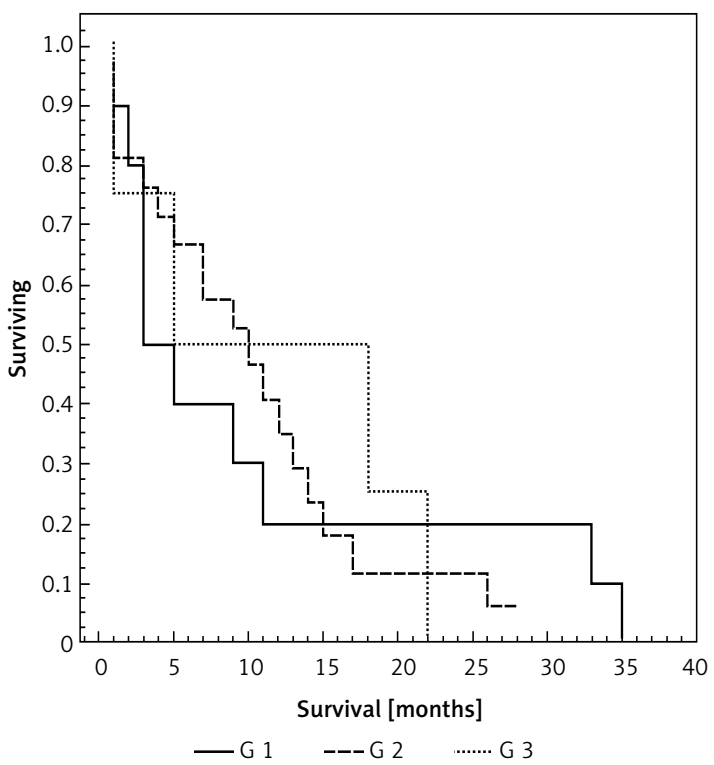

Figure 2. Kaplan-Meier curves were performed to assess the patient survival. A - Patient survival curves depending on the portal concentration of VEGF- $A_{165}$. Value $150.51 \mathrm{ng} / \mathrm{ml}$ is median concentration of VEGF- $A_{165}$ in portal blood among all patients $(p=0.12)$. B - Patient survival curves depending on the tumor differentiation (grade). Non-significant 
stimulates epithelial cell proliferation and migration, and inhibits apoptosis in order to form new blood vessels. Pancreatic cancer cells stimulated by some extrinsic factors, mainly by hypoxia, produce VEGF. Expression of VEGF is regulated mainly by hypoxia inducible factor (HIF-1) signaling. The PDAC shows high levels of hypoxia; in these tumors the level of HIF-1 expression is correlated with prognosis and with expression of VEGF [15, 16]. The level of VEGF expression in PDAC pathological specimens is associated with the presence of metastases to the lymph nodes and liver [17, 18], grade and mean vascular density (MVD) [19] in tumor, although certain publications do not confirm it [20]. The serum VEGF/soluble VEGF-receptor 1 ratio is also an independent prognostic marker in pancreatic cancer patients [21].

According to the results of the European Study Group for Pancreatic Cancer (ESPAC), tumor grade is one of the independent prognostic factors. Pongprasobchai et al. claim that long-term survival in small $(\leq 2 \mathrm{~cm})$ pancreatic cancer depends on tumor differentiation, not the tumor size. Grade is the only independent factor predicting survival in small PDAC [22]. Some studies do not confirm tumor grading on the prognosis in PDAC [23]. This discrepancy in the literature may be explained by the subjective assessment of pathological specimens, concerning the tumor differentiation. The problem lies in the lack of a universal system of assessment in cases of PDAC. Most pathologists seem to use a subjective approach, instead of the WHO system [24].

In our study, 31 out of 36 specimens were assessed according to the WHO criteria in standard pathological assessment in the Department of Pathology. Each sample was assessed by two experienced pathologists. In 22 out of 36 cases, the tumor was completely excised and examined, and in 14 cases the pathological examination was based on the intraoperative tumor biopsy or fine needle biopsy obtained preoperatively. Only 5 out of 22 resected tumors were smaller than $2 \mathrm{~cm}$. None of the patients was in stage I of the disease. Therefore, our survival curves are biased by the nonhomogeneous group of mainly advanced tumors. The role of early detection of an early staged PDAC is pivotal for the survival rate after tumor resection. However, the chances for resectability of PDAC at the time of detection are $10-20 \%[2,3]$ and the probability that the tumor is at stage I are even lower. In our group there were no patients with stage I of the disease. In 14 patients the tumors were so advanced that our strategy was focused on relieving symptoms associated with tumor compression of adjacent organs such as jaundice and/or gastric outlet obstruction and/or pain by performing surgical bypass procedures.
In our study we found a correlation between cancer grade and portal VEGF-A ${ }_{165}$ concentration. The VEGF-A ${ }_{165}$ concentration has a linear correlation with tumor differentiation. According to the ESPAC data, the tumor characteristics have the biggest impact on the prognosis in PDAC. In the multivariate regression analysis high levels of VEGF-A $A_{165}$ were not associated with poor survival $(\mathrm{HR}=5.22,95 \% \mathrm{Cl}=-0.6457$ to 3.9513 , $p=0.16)$. To achieve statistical significance, a larger group of patients is required. We found no differences in survival rate associated with tumor grade (Figure 2 B). Most likely that was associated with the relatively small and non-homogeneous group of patients, at different stages of the disease. However, VEGF-A ${ }_{165}$ concentration may also be elevated by the surgical procedure itself, as one of the main VEGF sources is platelets $[25,26]$. According to Belizon et al., the highest VEGF-A $A_{165}$ concentration was noted on the third day after the surgery. On the first post-operative day, the VEGF-A ${ }_{165}$ levels were increased significantly in the group of open surgery and not increased significantly in the groups of laparoscopic procedures. Also, the plasma VEGF-A ${ }_{165}$ concentration was correlated with the incision length [27]. There are no studies describing the VEGF concentration changes during the surgical procedures. Almost all known studies describe the elevation of the VEGF level on the first to third postoperative day [27]. In our investigations we assumed that collecting the portal blood from the portal vein in the same step of the operations (within the first 45 min after skin incision) does not alter the portal VEGF-A ${ }_{165}$ concentration. We also measured the plasma VEGF-A $A_{165}$ level just before surgery, in the superior vena cava (sampled using the central line) in a randomly selected subgroup of our patients (19 out of 36) and correlated the levels with those measured in the portal vein. There was a strong positive correlation $(R=0.9, p<0.05)$ but those levels did not correlate with cancer grade, size and survival with statistical significance (full data not shown). Therefore, we could assume that there is very low probability that portal VEGF-A $A_{165}$ levels are biased by the surgical trauma in our study. None of the patients qualified for the study were receiving any anti-platelet agents or steroids that could alter the VEGF-A ${ }_{165}$ measurements and of course clotting before surgery. Measuring the VEGF-A $A_{165}$ level in portal blood intraoperatively could be a safe and fast method of acquiring a factor that is highly correlated with the grade of the tumor. According to other studies, tumor grade is associated with the prognostic survival rate. However, in our study, probably due to the relatively small group of patients, VEGF-A ${ }_{165}$ levels were not clearly associated with prognosis. 
In our department we advocate palliative pancreaticoduodenectomies in all suitable cases. According to Lillemoe et al. pancreaticoduodenectomy performed as a palliative procedure has resulted in improved survival compared to bypass procedures [28]. Bockhorn et al. confirmed that although palliative resection results in elevated perioperative morbidity in comparison with surgical bypass, mortality is equal and survival is significantly prolonged [29]. The only contraindications for a radical procedure were: infiltration of the superior mesenteric artery, hepatic artery, celiac trunk, metastatic disease and advanced age. Infiltration of the portal vein was not a contraindication for pancreatoduodenectomy in our department. In 4 out of 22 patients pancreatoduodenectomy was performed, despite infiltration of the portal vein which was partially resected. We did not observe portal vein thrombosis after surgery.

According to Garcea et al. better survival rates could be achieved by employing selective criteria on operability [30], which was also confirmed by Yamada et al. in the case of periaortic lymph node metastasis [31]. Therefore, identifying the group of patients with the best chances for long-term survival, i.e. the lowest tumor grade, is feasible during an operation and could alter the treatment plan, encouraging the most radical surgery despite advanced tumor - palliative pancreatoduodenectomy. But that needs further investigations with long-term follow-up.

\section{References}

1. Jemal A, Siegel R, Ward E. Cancer statistics, 2006. CA Cancer J Clin 2006; 56: 106-30.

2. Nitecki SS, Sarr MG, Colby TV. Long-term survival after resectionfor ductal adenocarcinoma of the pancreas. Is it really improving ? Ann Surg1995; 221: 59-66.

3. Alexakis N, Halloran C, Raratz M. Current standards of surgery for pancreatic cancer. Br J Surg 2004; 91: 1410-27.

4. Wan C, Shen Y, Yang T, et al. Diagnostic value of microRNA for pancreatic cancer: a meta-analysis. Arch Med Sci 2012; 8: 749-55.

5. Folkman J. Tumor angiogenesis: therapeutic implications. N Engl J Med 1971; 285: 1182-6.

6. Hoeben A, Landuyt B, Highley MS. Vascular endothelial growth factor and angiogenesis. Pharmacol Rev 2004, 56: 549-80.

7. Hoeben A, Landuyt B, Highley MS, Wildiers H, Van Oosterom A, De Bruijn EA. Vascular endothelialgrowth factor and angiogenesis. Pharmacol Rev 2004; 56: 549-80.

8. Ferrara N, Davis-Smyth T. The biology of vascular endothelial growth factor. Endocrine Rev 1997; 18: 4-25.

9. Shibuya M. Vascular endothelial growth factor-dependent and -independent regulation of angiogenesis. BMB Reports 2008; 41: 278-86.

10. Tang RF, Wang SX, Peng L. Expression of vascular endothelial growth factors $A$ and $C$ in human pancreatic cancer. World J Gastroenterol 2006; 12: 280-6.

11. Chang YT, Chang MC, Wei SC, et al. Serum vascular endothelial growth factor/soluble vascular endothelial growth factor receptor 1 ratio is an independent prognostic marker in pancreatic cancer. Pancreas 2008; 37: 145-50.

12. Sandblom G, Granroth S, Rasmussen IC. TPS, CA 19-9, VEGF-A, and CEA as diagnostic and prognostic factors in patients with mass lesions in the pancreatic head. Ups J Med Sci 2008; 113: 57-64.

13. Ai KX, Lu LY, Huang XY, Chen W, Zhang HZ. Prognostic significance of S100A4 and vascular endothelial growth factor expression in pancreatic cancer. World J Gastroenterol 2008; 14: 1931-5.

14. Hotz HG, Hines OJ, Masood R. VEGF antisense therapy inhibits growth and improves survival in experimental pancreatic cancer. Surgery 2005; 137: 192-9.

15. Hoffmann AC, Mori R, Vallbohmer D, et al. High expression of HIF1a is a predictor of clinical outcome in patients with pancreatic ductal adenocarcinomas and correlated to PDGFA, VEGF, and bFGF. Neoplasia 2008; 10: 674-9.

16. Shibaji T, Nagao M, Ikeda N, et al. Prognostic significance of HIF-1 alpha overexpression in human pancreatic cancer. Anticancer Res 2003; 23: 4721-7.

17. Niedergethmann M, Hildenbrand R, Wostbrock B, et al. High expression of vascular endothelial growth factor predicts early recurrence and poor prognosis after curative resection for ductal adenocarcinoma of the pancreas. Pancreas 2002; 25: 122-9.

18. Karayiannakis AJ, Bolanaki H, Syrigos KN, et al. Serum vascular endothelial growth factor levels in pancreatic cancer patients correlate with advanced and metastatic disease and poor prognosis. Cancer Lett 2003; 194: 119-24.

19. Seo Y, Baba H, Fukuda T, Takashima M, Sugimachi K. High expression of vascular endothelial growth factor is associated with liver metastasis and a poor prognosis for patients with ductal pancreatic adenocarcinoma. Cancer 2000; 88: 2239-45.

20. Lim YJ, Lee JK, Park CK, et al. Prognostic value of VEGF in human pancreatic ductal adenocarcinoma. Korean J Intern Med 2004; 19: 10-4.

21. Chang YT, Chang MC, Wei SC, et al. Serum vascular endothelial growth factor/soluble vascular endothelial growth factor receptor 1 ratio is an independent prognostic marker in pancreatic cancer. Pancreas 2008; 37: 145-50.

22. Pongprasobchai S, Pannala R, Smyrk TC. Long-term survival and prognostic indicators in small $(\leq 2 \mathrm{~cm})$ pancreatic cancer. Pancreatology 2008; 8: 587-92.

23. Ahmad NA, Lewis JD, Ginsberg GG. Long term survival after pancreatic resection for pancreatic adenocarcinoma. Am J Gastroenterolgy 2001; 96: 2609-15.

24. Adsay NV, Bastruk O, Bonnett M, et al. A proposal for a new and more practical grading scheme for pancreatic ductal adenocarcinoma. Am J Surg Pathol 2005; 29: 724-33.

25. Gunsilius E, Petzer A, Stockhammer G. Thrombocytes are the major source for soluble vascular endothelial growth factor in peripheral blood. Oncolology 2000; 58: 169-74.

26. Banks RE, Forbes MA, Kinsey SE. Release of the angiogenic cytokine vascular endothelial growth factor (VEGF) from platelets - significance for VEGF measurements and cancer biology. Br J Cancer 1998; 77: 956-64.

27. Belizon A, Balik E, Feingold DL. Major abdominal surgery increases plasma levels of vascular endothelial growth factor. Open more so than minimally invasive methods. Ann Surg 2006; 244: 792-8. 
28. Sohn TA, Nakeeb A, Sauter PK. Pancreaticoduodenectomy. Does it have a role in the palliation of pancreatic cancer. Ann Surg 1996; 223: 718-25.

29. Bockhorn M, Cataldegirmen G, Kutup A, et al. Crossing the Rubicon: when pancreatic resection with curative intent ends in an R2 status. Impact of "desmoplastic pseudo-pancreatitis" and anatomical site of irresectability. Ann Surg Oncol 2009; 16: 1212-21.

30. Garcea G, Dennison AR, Ong SL, et al. Tumor characteris tics predictive of surviaval following resection for ductal adenocarcinoma of th head of pancreas. EJSO 2007; 33 : 892-7.

31. Yamada S, Nakao A, Fuji T, et al. Pancreatic cancer with paraaortic lymph node metastasis: a contraindication for radical surgery ? Pancreas 2009; 38: e13-7. 